\title{
Hmga2 is required for canonical WNT signaling during lung development
}

Indrabahadur Singh ${ }^{1}$, Aditi Mehta' ${ }^{1}$ Adriana Contreras ${ }^{1}$, Thomas Boettger ${ }^{2}$, Gianni Carraro ${ }^{3}$, Matthew Wheeler ${ }^{2}$, Hector A Cabrera-Fuentes ${ }^{4,5}$, Saverio Bellusci ${ }^{3}$, Werner Seeger ${ }^{6}$, Thomas Braun ${ }^{2}$ and Guillermo Barreto ${ }^{1^{*}}$

\begin{abstract}
Background: The high-mobility-group (HMG) proteins are the most abundant non-histone chromatin-associated proteins. HMG proteins are present at high levels in various undifferentiated tissues during embryonic development and their levels are strongly reduced in the corresponding adult tissues, where they have been implicated in maintaining and activating stem/progenitor cells. Here we deciphered the role of the high-mobility-group AT-hook protein 2 (HMGA2) during lung development by analyzing the lung of Hmga2-deficient mice $\left(\mathrm{Hmga2}^{-1-}\right)$.

Results: We found that Hmga2 is expressed in the mouse embryonic lung at the distal airways. Analysis of $\mathrm{Hmga2}^{-/-}$mice showed that Hmga2 is required for proper cell proliferation and distal epithelium differentiation during embryonic lung development. Hmga2 knockout led to enhanced canonical WNT signaling due to an increased expression of secreted WNT glycoproteins Wnt2b, Wnt7b and Wnt11 as well as a reduction of the WNT signaling antagonizing proteins GATA-binding protein 6 and frizzled homolog 2. Analysis of siRNA-mediated loss-of-function experiments in embryonic lung explant culture confirmed the role of Hmga2 as a key regulator of distal lung epithelium differentiation and supported the causal involvement of enhanced canonical WNT signaling in mediating the effect of Hmga2-loss-of-fuction. Finally, we found that HMGA2 directly regulates Gata6 and thereby modulates Fzd2 expression.
\end{abstract}

Conclusions: Our results support that Hmga2 regulates canonical WNT signaling at different points of the pathway. Increased expression of the secreted WNT glycoproteins might explain a paracrine effect by which Hmga2-knockout enhanced cell proliferation in the mesenchyme of the developing lung. In addition, HMGA2-mediated direct regulation of Gata6 is crucial for fine-tuning the activity of WNT signaling in the airway epithelium. Our results are the starting point for future studies investigating the relevance of Hmga2-mediated regulation of WNT signaling in the adult lung within the context of proper balance between differentiation and self-renewal of lung stem/ progenitor cells during lung regeneration in both homeostatic turnover and repair after injury.

Keywords: Branching morphogenesis, HMGA2, GATA6, Lung development, WNT signaling

\section{Background}

The mouse lung arises from the anterior endoderm and forms during five overlapping phases of lung development: embryonic (embryonic days post coitum (E) 9 to 12.5), pseudoglandular (E12.5 to E16.5), canalicular (E16.5 to E17.5), saccular (E17.5 to post-natal day (P) 5) and alveolar (P5 to P28) [1-3]. At the end of the embryonic phase, primary and secondary lung buds formation has taken place and the embryonic lung consists of one

\footnotetext{
* Correspondence: guillermo.barreto@mpi-bn.mpg.de

${ }^{1}$ LOEWE Research Group Lung Cancer Epigenetic, Max-Planck-Institute for Heart and Lung Research, Parkstraße 1, 61231 Bad Nauheim, Germany
} Full list of author information is available at the end of the article left lobe and four right lobes. From E10.5 to E16.5, the epithelium undergoes branching morphogenesis to form the respiratory (bronchial) tree. In parallel to branching morphogenesis, the airway epithelium differentiates from a morphologically uniform cell population to different specialized cell types, thereby establishing a proximaldistal axis in the developing lung. However, most of the differentiation occurs in the canalicular and saccular phases (E16.5 to P5). The primitive lung epithelium co-expresses several lineage markers including Clara cell-specific $10 \mathrm{kDa}$ protein (Scgb1a1, also CC10) and surfactant-associated protein C (Sftpc, also SP-C). Later in gestation (E16.5 onwards), Scgb1a1 is a marker

\section{Biomed Central}


for the proximal epithelium, whereas Sftpc expression defines the distal epithelium. In the adult lung these markers are characteristic of distinct cell lineages, Scgb1a1 of Clara cells and Sftpc of alveolar type II cells. Only specific progenitor cells in the adult lung, bronchioalveolar stem cells (BASCs), co-express Scgb1a1 and Sftpc [4].

Several evolutionarily conserved signaling pathways have been implicated in different phases of embryonic lung development. In particular, members of the fibroblast growth factor, bone morphogenetic protein, hedgehog/Gli, epidermal growth factor and wingless secreted glycoprotein (WNT) families have been implicated in lung morphogenesis and epithelial differentiation [2,5-7]. In addition, a well-organized and balanced interplay between these signaling pathways and key transcription factors of lung development, including NK2 homeobox 1 (also known as thyroid transcription factor 1), forkhead box protein A2 (also known as hepatocyte nuclear factor 3-beta) and GATA6, is required for proper lung formation [2,3,7]. GATA6 is the only member of the GATA family of zinc finger transcription factors that is expressed in the distal epithelium of the developing lung $[8,9]$. GATA6 is essential for branching morphogenesis and regulates differentiation of distal lung epithelium $[9,10]$. Moreover, GATA6 has been implicated in blocking WNT signaling to control the balance between BASC expansion and lung epithelial differentiation required for both lung development and regeneration [11].

High mobility group AT-hook protein 2 (HMGA2) is a transcription regulator belonging to the family of HMG proteins. HMG proteins are the most abundant nonhistone chromatin-associated proteins and regulate gene expression by altering chromatin structure and recruiting other proteins to the transcription regulatory complex [12]. HMGA2 is present at high levels in various undifferentiated tissues during embryonic development and its levels are strongly reduced in the corresponding adult tissues [12,13]. In addition, Hmga 2 expression in adult organs has been implicated in maintaining and activating stem/progenitor cells in different tissues $[14,15]$. Here, we show that Hmga2 mRNA levels are high during early stages of lung development, in which cells are undifferentiated, and become reduced and restricted to the distal airways as lung development progresses, coincident with cell differentiation. Analysis of the lung of Hmga2-knockout (KO) mice [16] revealed enhanced canonical WNT signaling that led to increased cell proliferation, increased number of progenitor cells and reduced differentiation of the distal airway epithelium. Using a lung explants culture system, we confirmed the causal involvement of WNT signaling mediating the effect of Hmga2-loss-of-function (LOF) and showed that Hmga2 is required for proper branching morphogenesis during the formation of the bronchial tree. Furthermore, we showed that Hmga2 regulates canonical WNT signaling at different points of the pathway. Increased expression of the secreted WNT glycoproteins might explain a paracrine effect by which Hmga2-KO enhanced cell proliferation in the mesenchyme of the developing lung. In addition, HMGA2-mediated direct regulation of Gata6 is crucial for fine-tuning the activity of WNT signaling in the airway epithelium.

\section{Results}

Hmga2 is expressed in the mouse embryonic lung at the distal airways

To verify that Hmga2 is expressed during lung development, quantitative reverse transcription PCR (qRT-PCR) expression analysis was performed (Figure 1A). Hmga2 transcript was detected at E11.5, when the primary lung buds have evaginated from the foregut and secondary buds are forming as outgrowths from the primary lung buds. Hmga 2 expression progressively decreased during the pseudoglandular stages of lung development (E12.5 to E16.5). Between the canalicular (E16.5 to E17.5) and initial saccular stages (E17.5 to E18.5), the levels of Hmga 2 transcript increased again. Later in gestation (saccular stages, E18.5 to P5), Hmga2 expression was further reduced and reached a basal level of expression that was maintained through the alveolar phase (P5 to P28). Thus, Hmga 2 mRNA levels were high during early stages of lung development, in which cells are undifferentiated, and decreased as lung development progressed, coincident with cell differentiation.

In situ hybridization expression pattern analysis in the embryonic lung at E12.5 (Figure 1B), when branching morphogenesis of the lung bud is proceeding rapidly to establish the future bronchial tree, revealed that Hmga2 is ubiquitously expressed with higher levels of expression at the tips of the growing lung buds. Interestingly, Hmga 2 expression became restricted to the distal lung endoderm at E14.5. Consistently, immunostaining on sections of the embryonic lung at E14.5 (Figure 1C) supported the presence of HMGA2 in cells of the distal lung endoderm. Co-staining with an antibody specific for the nuclear envelope protein lamin B1 (LMNB1) demonstrated the nuclear localization of HMGA2. The observed expression patterns in embryonic lung suggest a role for HMGA2 in epithelial differentiation.

\section{Hmga2 is required for proper differentiation of the distal epithelium during lung development}

To determine the role of Hmga2 during lung development, we analyzed the embryonic lung of Hmga2-deficient mice $\left(\mathrm{Hmga2}^{-/}\right)$[16]. At E18.5, when the bronchial tree is complete and the lung tissues are differentiating into different cell types that will constitute the lung after birth, 

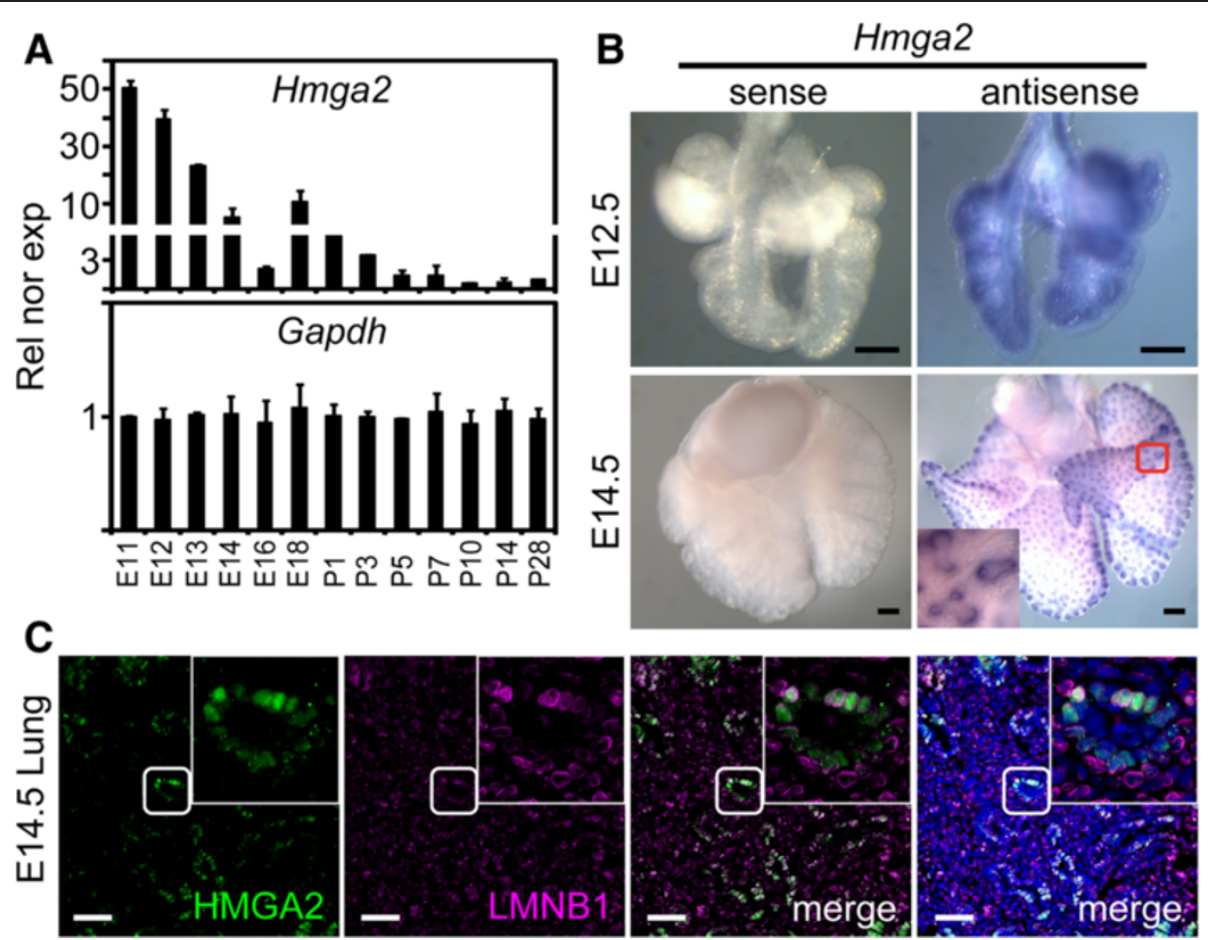

Figure $1 \mathrm{Hmga2}$ is expressed in the mouse embryonic lung at the distal airways. (A) Hmga2 is expressed during embryonic lung development. Hmga2 and Gapdh expression was monitored by quantitative RT-PCR in mouse embryonic lung at different days post coitum (E11.5 to E18.5) and in mouse lung at different days after birth (P1 to P28). Rel nor exp, relative expression normalized to Tuba1a. Error bars show the SEM $(n=4)$. (B) Hmga2 is expressed in the mouse embryonic lung at the distal airways. Hmga2 mRNA was detected in mouse embryonic lung at E12.5 and E14.5 by in situ hybridization using an Hmga2-specific antisense RNA probe. Sense probe, negative control. Scale bars, $200 \mu$ m. Square shows details at higher magnification. (C) HMGA2 localized in the cell nuclei of the embryonic lung. Fluorescence microscopy of embryonic lung sections (E14.5) after double immunostaining using HMGA2- and LMNB1-specific antibodies. Nuclear staining with DAPI (blue). Squares as in B. Scale bars, $40 \mu \mathrm{m}$.

Hmga2-KO resulted in a reduced body weight and lungto-body-wet-weight ratio (Additional file 1: Figures S1A and Figure 2A). Macroscopically, the embryonic lung of Hmga2-KO mice at E18.5 had four lobes on the right side and one lobe on the left, indicating that the earliest events during lung development, specification of pulmonary endoderm and primary branching morphogenesis, occurred normally and do not require Hmga2. However, histological analysis of the embryonic lung at this stage (Figure 2B) revealed a marked increase of cell density in the $\mathrm{Hmga2}^{-/-}$mice when compared with the wild-type (WT) mice, suggesting increased cell proliferation. Furthermore, immunostaining on sections of embryonic lung (Figure 2C) using antibodies specific for the epithelial marker pan-cytokeratin (KRT) and the mesenchymal marker vimentin (VIM) showed a broader mesenchyme and an irregularly shaped epithelium in Hmga2-KO mice when compared to the WT mice. This suggests an expansion of the mesenchyme at the expense of the epithelium. Consistent with this observation, quantification of KRTpositive cells (Figure 2D, left) showed a decrease from $61.7 \%$ to $29.5 \%(P<0.001 ; n=3)$, whereas the number of VIM-positive cells (right) increased from $35.7 \%$ to $46.3 \%$
$(P<0.001 ; n=3)$ in Hmga2-KO mice when compared to the WT mice. Immunostaining on sections of embryonic lung showed increased levels of proliferation markers, specifically proliferating cell nuclear antigen (PCNA) and antigen identified by monoclonal antibody Ki 67 (MKI67) (Figure 2E and Additional file 1: Figure S1C), in both the epithelium and the mesenchyme of the Hmga2-KO mice, as shown by co-staining with antibodies specific for KRT (left) or VIM (right), supporting our interpretation of the histological analysis. However, quantification of our results (Figure 2D) showed more prominent cell proliferation in the mesenchyme (right), from $3.9 \%$ to $43.3 \%(P<0.001$; $n=3$ ) when compared to the epithelium (left), from $8 \%$ to $23.6 \%(P<0.001 ; n=3)$. To confirm increased cell proliferation in the embryonic lung after Hmga2-KO, we analyzed the expression of cell-cycle progression markers cyclin E1, E2 and D2 (Figure 2F). Expression of these genes increased after Hmga2-KO when compared to WT mice, demonstrating elevated cell proliferation.

In addition to cell proliferation, programmed cell death is an important process involved in lung development [17]. Thus, we performed co-staining on sections of embryonic lung (Figure 3A) using antibodies specific 


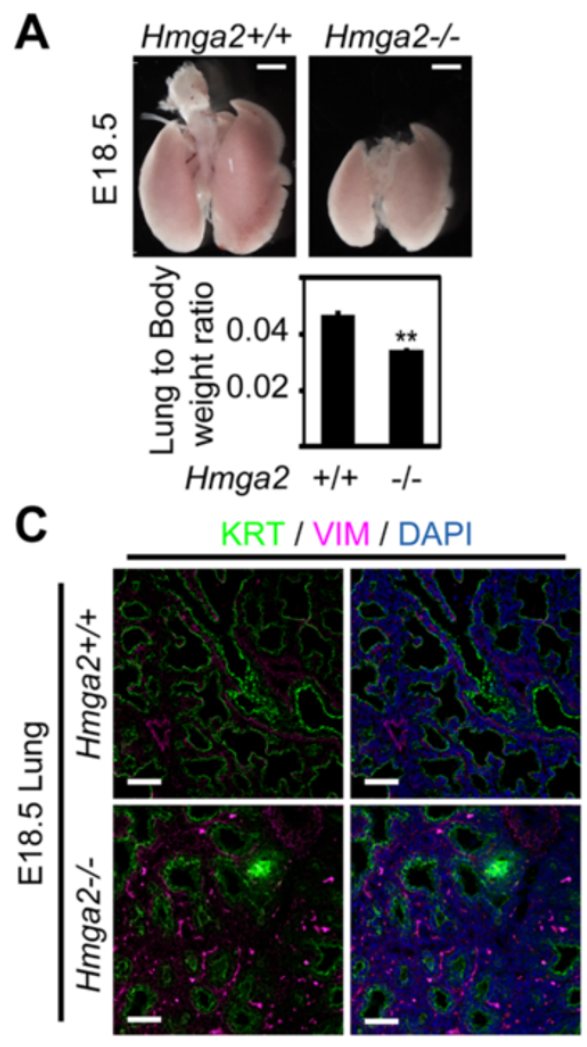

E

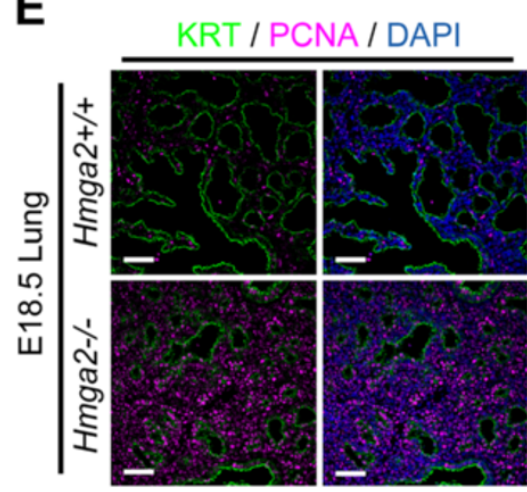

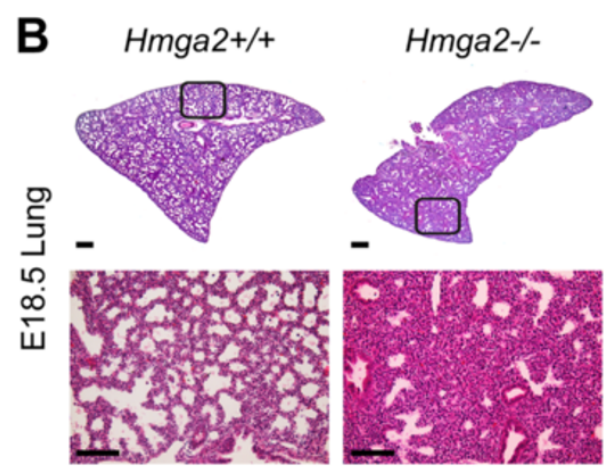

D
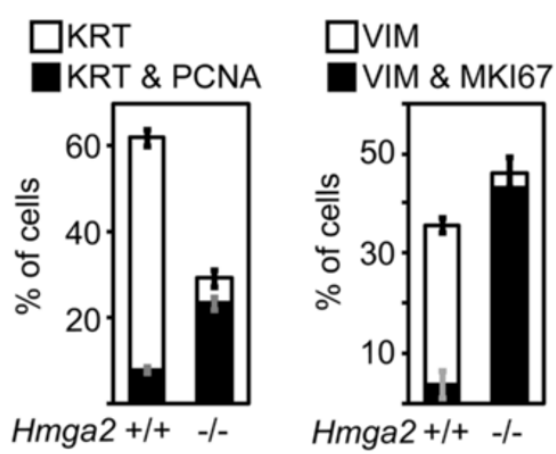

$\mathbf{F}$
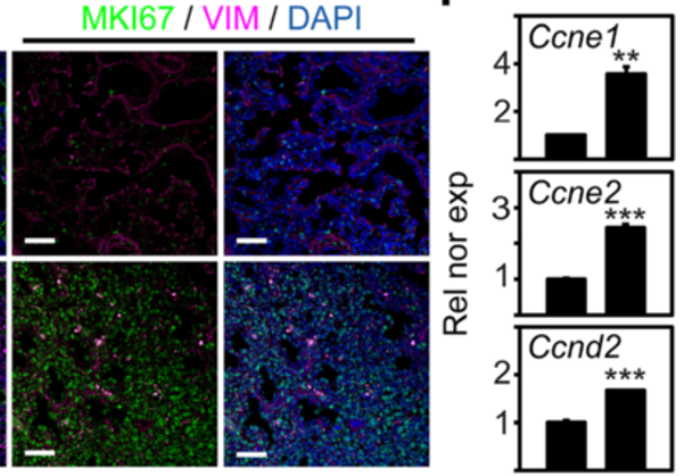

Hmga2 +/+ - /-

Figure $2 \mathrm{Hmga2}^{-/-}$mice embryonic lung showed increased cell proliferation. (A) Lungs of Hmga2-KO (-/-) embryo were smaller (top) and showed a reduced lung-to-body-wet-weight ratio (bottom) when compared to WT (+/+) embryos at E18.5. Scale bars, $1 \mathrm{~mm}$. Error bars show the SEM $(n=6)$. ${ }^{* *} P<0.001 ;{ }^{* *} P<0.01 ;{ }^{*} P<0.05$ after one-way analysis of variance. (B) Representative of histological analysis using hematoxylin and eosin stain on sections of WT and Hmga2-KO embryonic lung at E18.5. Squares are shown at the bottom at higher magnification. Scale bars, 100 m. (C) Sections of embryonic lung (E18.5) of WT and Hmga2-KO mice were analyzed by confocal microscopy after double immunostaining using KRT- and VIM-specific antibodies. Nuclear staining with DAPI (blue). Scale bars, 40 m. (D) Quantification of proliferating (PCNA- or MKI67-positive) epithelial (KRT) and mesenchymal (VIM) cells showed increased proliferation in both tissues of Hmga2-KO embryonic lung. Sections of embryonic lung (E18.5) of WT and Hmga2-KO mice were treated as in E and used for quantification. Error bars show the SEM $(n=3)$. (E) Sections of embryonic lung (E18.5) of WT and Hmga2-KO mice were analyzed as in C using (left) KRT- and PCNA-specific antibodies or (right) VIM- and MKI67-specific antibodies. Nuclear staining with DAPI (blue). Scale bars, 40 m. (F) Hmga2-KO enhanced expression of cell-cycle progression markers. Expression analysis of the indicated genes by quantitative RT-PCR in E18.5 lung of WT and Hmga2-KO mice. Rel nor exp, relative expression normalized to Tuba1a. Error bars show the SEM ( $n=4)$. Asterisks as in A.

for the active form of the apoptosis-related cysteine peptidase, cleaved caspase 3 (clCASP3) and KRT (left) or the mesenchymal marker smooth muscle actin alpha 2
(ACTA2, right). Quantification of clCASP3-positive cells (Figure 3B) showed that whereas the level of apoptosis in the mesenchyme was not significantly affected after 


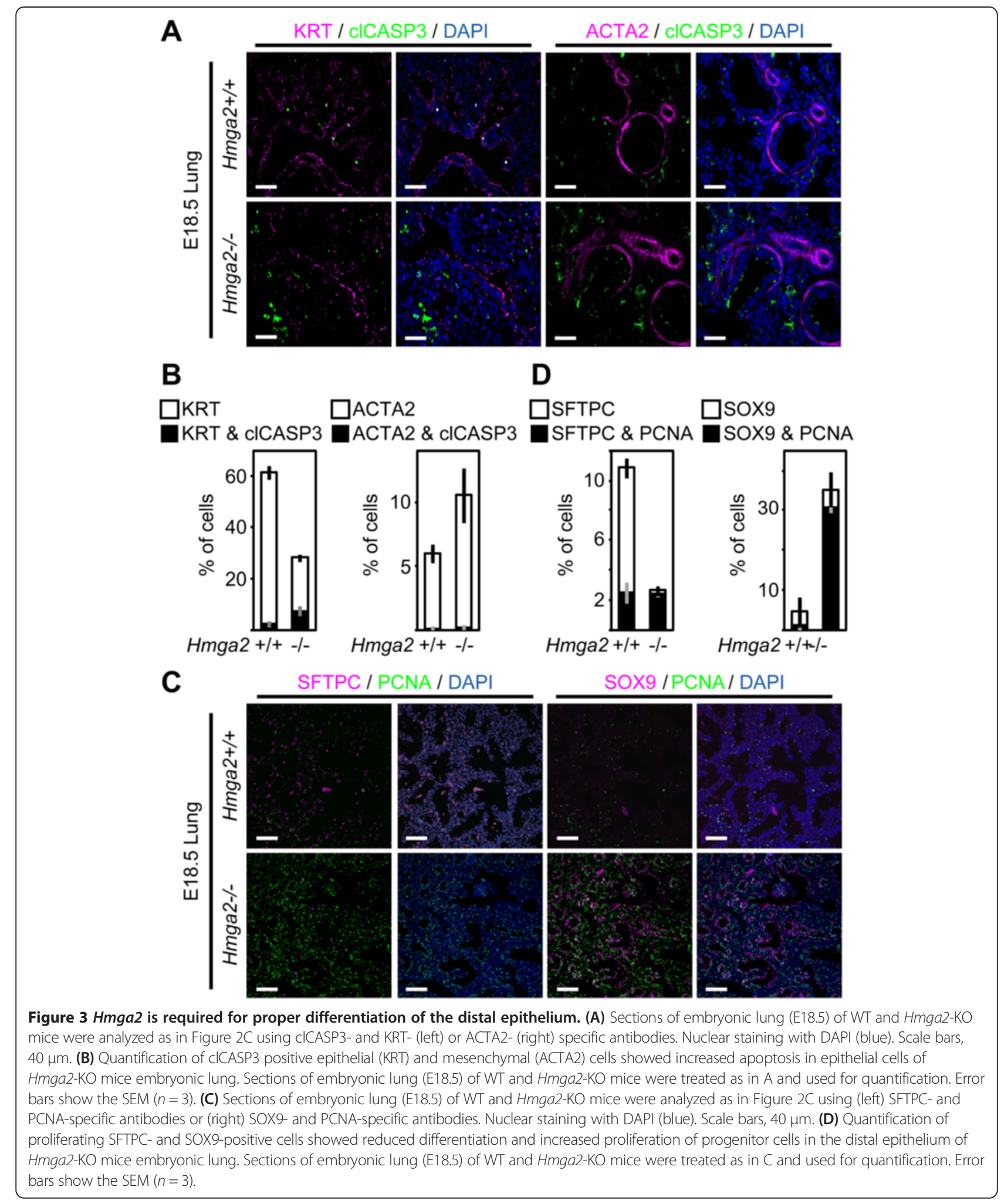

Hmga2-KO (0.2\% in the WT versus $0.3 \%$ in the $\mathrm{KO})$, it increased in the lung epithelium of $\mathrm{Hmga2}^{-/-}$mice from $2.7 \%$ to $7.5 \%(P<0.01 ; n=3)$, supporting the reduction of epithelium when compared with WT mice. However, since this increase of apoptosis cannot account for the drastic reduction of lung epithelium after Hmga2-KO, we examined lung epithelium differentiation by analyzing the expression of proximal, Scgbla1, and distal, 
Sftpc, epithelial markers in the embryonic lung of WT and Hmga 2-KO mice (Additional file 1: Figure S1B). Whereas Scgb1a1 expression did not change significantly, Sftpc expression was reduced after Hmga2-KO, suggesting defects in distal epithelium differentiation. These defects were confirmed by immunostaining on sections of embryonic lung (Figure 3C,D, left) that showed reduced numbers of SFTPC-positive cells from $10.8 \%$ to $2.7 \%(P<0.001 ; n=3)$ after Hmga2-KO when compared with the WT mice. These results were confirmed in lung explants after siRNAmediated Hmga2 knockdown (KD) (Additional file 2: Figure S2A). To further investigate these results, we performed immunostaining on sections of embryonic lung using antibodies specific for the distal epithelium progenitor cell marker sex determining region Y-box 9 (SOX9) and PCNA (Figure 3C,D, right). The number of SOX9positive cells increased from $3.8 \%$ to $35.3 \%(P<0.001$; $n=3)$ after Hmga2-KO when compared with WT mice. Interestingly, most of the SOX9-positive cells in the Hmga2-KO lung were proliferating as shown by PCNA co-staining. To summarize, Hmga2-KO increased cell proliferation more significantly in the mesenchyme of the embryonic lung, increased apoptosis in the epithelium, and reduced differentiation of the distal airway epithelium by altering the balance between progenitor cell renewal and differentiation. Taking together, our data support that Hmga 2 is required for proper distal epithelium differentiation.

\section{Hmga2 knockout led to enhanced canonical WNT signaling}

Comparison of $\mathrm{Hmga2}^{-/-}$with $\mathrm{Hmga2}^{+/+}$mice by Affymetrix microarray-based expression analysis of embryonic lung (Figure 4A) revealed an increased expression of cellcycle-related genes, confirming our immunohistological results. We found that transcripts of genes that are either targets or positive regulators of canonical WNT signaling pathway increased in the embryonic lung of Hmga2-KO mice. Furthermore, expression of negative regulators of canonical WNT signaling was reduced in Hmga2-KO mice. These results indicate enhancement of canonical WNT signaling after Hmga2-KO that was further validated by increased activity of the beta-catenin/T-cell factor (TCF)/lymphoid enhancer factor (LEF) WNT-reporter (BAT-GAL) in embryonic lung of the BAT-GAL:Hmga2 ${ }^{-/-}$ double transgenic mice when compared to BAT-GAL: $\mathrm{Hmga2}^{+/+}$(Figure 4B). Western blot analysis of embryonic lung protein extracts (Figure 4C) showed increased levels of activated-beta-catenin $(\mathrm{ABC})$ and phosphorylation of the WNT co-receptor low-density lipoprotein receptor-related protein 6 (LRP6) [18-20] after Hmga2-KO, confirming enhancement of canonical WNT signaling. However, betacatenin (CTNNB1) has a dual role in both WNT signaling and cell adhesion processes. To confirm enhanced WNT signaling after Hmga2-KO, we analyzed the expression of canonical WNT targets, Axin2, Mycn (v-myc myelocytomatosis viral related oncogene), Fgfr2 (fibroblast growth factor receptor 2) and Bmp4 (bone morphogenetic protein 4) in the embryonic lung (Figure 4D). Expression of canonical WNT targets increased after Hmga2-KO when compared to WT mice, demonstrating elevated canonical WNT signaling. Western blot analysis of protein extracts from embryonic lung (Figure 4E) showed increased levels of canonical WNT targets after Hmga2-KO, confirming our expression analysis. Our results in the embryonic lung of $\mathrm{Hmga2}^{-/-}$mice were supported by siRNA-mediated Hmga 2 depletion in the mouse lung epithelial cell line MLE-12 (Additional file 3: Figure S3), which increased the basal transcription of a co-transfected WNT-responsive luciferase reporter (p3LEF-LUC) more than twofold when compared to control siRNA (siCtrl)-transfected cells. Interestingly, forced expression of Hmga2 reduced the basal transcription of $p 3 L E F-L U C$, thereby supporting an Hmga2-mediated negative regulation of WNT signaling.

Enhanced canonical WNT signaling is related to cell proliferation [21-23], correlating with our histological and molecular characterization of the Hmga2-KO embryonic lung. Nevertheless, we observed increased cell proliferation in the mesenchyme of embryonic lung after Hmga2-KO, although Hmga2 expression is restricted to the distal epithelium of the embryonic lung. A plausible explanation for these two observations could be that enhanced WNT signaling after Hmga2-KO is induced in part by diffusible positive regulators of canonical WNT signaling. Indeed, our Affymetrix microarray-based expression analysis (Figure 4A) showed elevated expression of Wnt11, Wnt7b and Wnt $2 b$ in embryonic lung of $\mathrm{Hmga2}^{-/-}$mice when compared to Hmga2 ${ }^{+/+}$. These results were confirmed by qRT-PCR-based expression analysis (Figure 4F). Furthermore, immunostaining on sections of embryonic lung using CTNNB1-specific antibody (Figure 4G) showed increased translocation of CTNNB1 from the cytoplasm into the nucleus in cells of both the epithelium and the mesenchyme after Hmga2$\mathrm{KO}$, demonstrating elevated canonical WNT signaling in both tissues and explaining hyperproliferation and expansion of the mesenchyme after Hmga2-KO.

To confirm the causal involvement of canonical WNT signaling in mediating the effect of Hmga2-LOF, we used an organ explant culture system that mimics the normal embryonic lung development (Additional file 4: Figure S4A,B) [24]. Depletion of Hmga2 in these organ explants by siRNA treatment (Additional file 4: Figure S4C,D) led to a marked impairment in branching morphogenesis when compared to explants exposed to control siRNA (siCtrl) (Figure 5A). These defects during the formation of the bronchial and respiratory tree after Hmga2-KD were validated by quantification of the total number of 

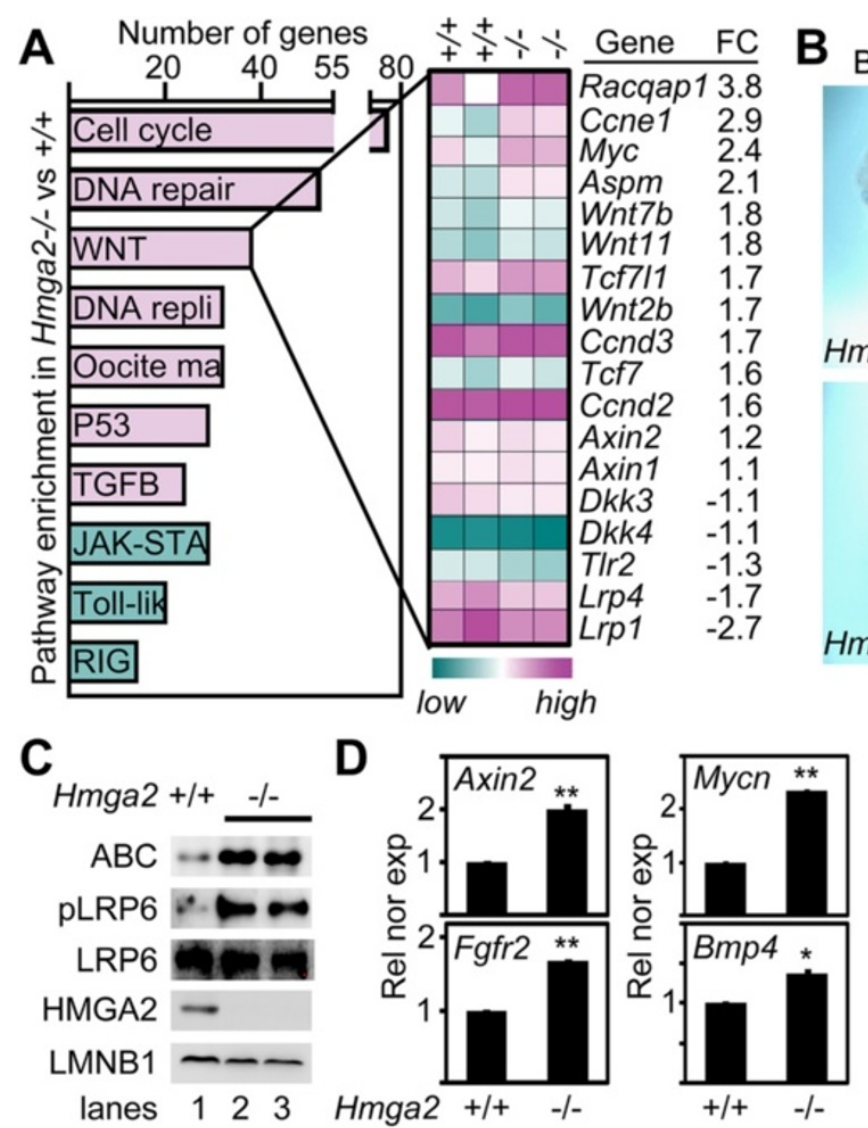

$\mathbf{E}$
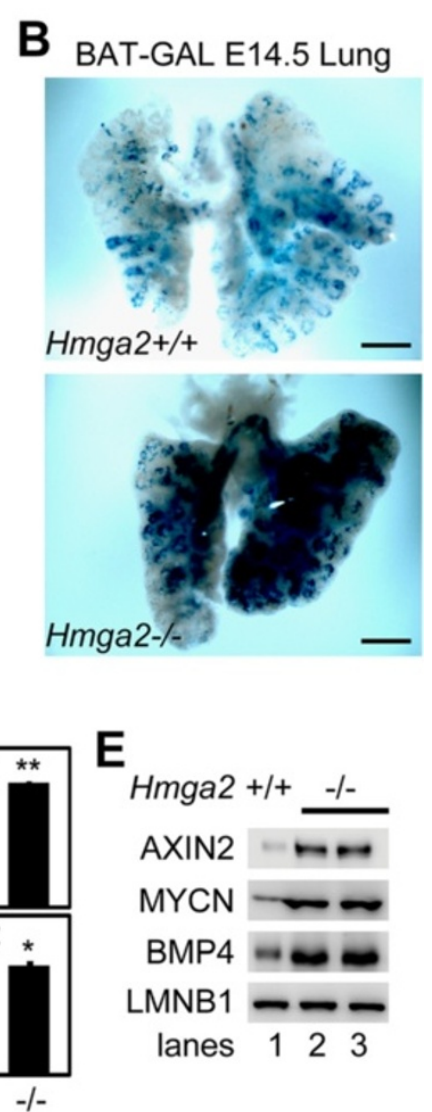

Hmga2- $\%$
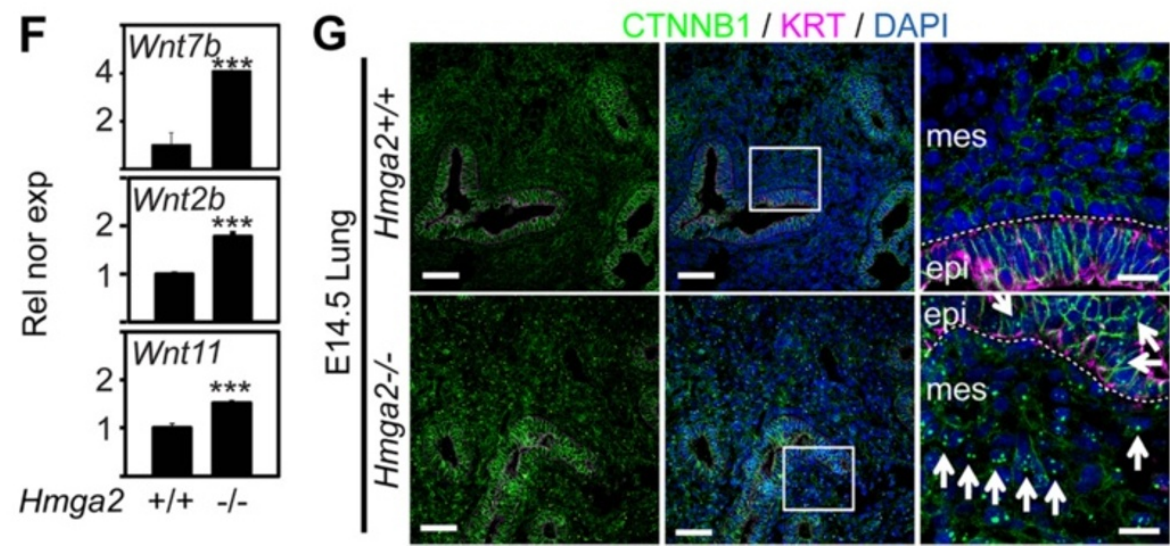

Figure $4 \mathrm{Hmga2}^{-/-}$mice embryonic lung showed enhanced canonical WNT signaling. (A) Enriched cellular pathways $(P<0.05)$ after Affymetrix microarray-based transcriptome analysis of $\mathrm{Hmga2}^{-/-}$E18.5 lung when compared to Hmga2 ${ }^{+/+}$. Left, Kyoto Encyclopedia of Genes and Genomes pathway analysis performed using DAVID bioinformatics tool. Green, reduced expression (fold change <1.2); magenta, increased expression (fold change $\geq 1.2$ ); repli, replication; ma, maturation; JAK-STA, Jak-STAT signaling; Toll lik, Toll-like-receptor signaling; RIG, RIG-like-receptor signaling. Right, Heat map was done using DNAStar Arraystar 11.0 and represents expression of genes involved in the WNT signaling pathway in Hmga2 ${ }^{+/+}$and Hmag2 $2^{-/-}$E18.5 lung. Green, low expression; magenta, high expression; FC, fold change. (B) Hmga2-KO enhanced beta-catenin/TCF/LEF reporter activity in E14.5 lungs of the BAT-GAL transgenic mice. Representative $(n=5)$ BAT-GAL staining (blue color) shows activated WNT signaling. Scale bars, 2 mm. (C) Hmga2-KO increased levels of activated-beta-catenin (ABC) and phosphorylated LRP6 (pLRP6). Protein extracts from WT or Hmga2-KO E18.5 lungs were analyzed by western blot using the indicated antibodies. Lanes 2 and 3 are biological duplicates. (D) Hmga2-KO enhanced expression of canonical WNT pathway markers. Expression analysis of the indicated genes as in Figure 2F. (E) Hmga2-KO increased protein levels of canonical WNT pathway markers. Protein extracts were analyzed as in C using the indicated antibodies. (F) Hmga2-KO enhanced expression of secreted WNT glycoprotein genes Wnt7b, Wnt2b and Wnt11. Expression analysis as in Figure 2F. (G) Hmga2-KO increased nuclear localization of CTNNB1 in cells of the epithelium (epi) and the mesenchyme (mes) of the embryonic lung. Sections of E14.5 lung of WT and Hmga2-KO mice were analyzed as in Figure 2C using KRT- and CTNNB1- specific antibodies. Square shows details at higher magnification on the right. Nuclear staining with DAPI (blue). Arrows indicate nuclear CTNNB1. Dashed line shows epithelium-mesenchyme-boundary. Scale bars, 40 and $10 \mu \mathrm{m}$ (right). 
A
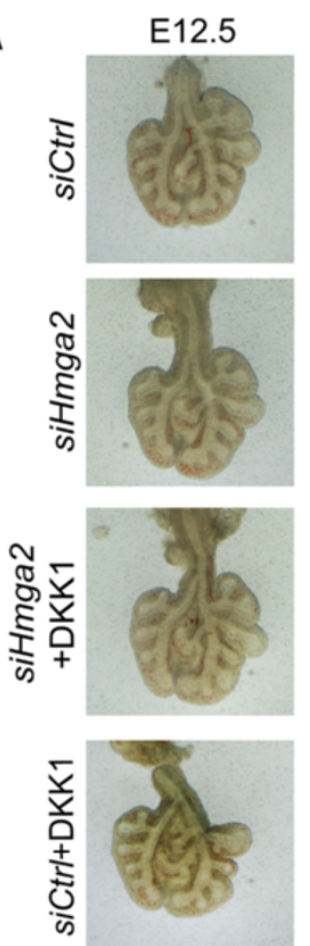

B
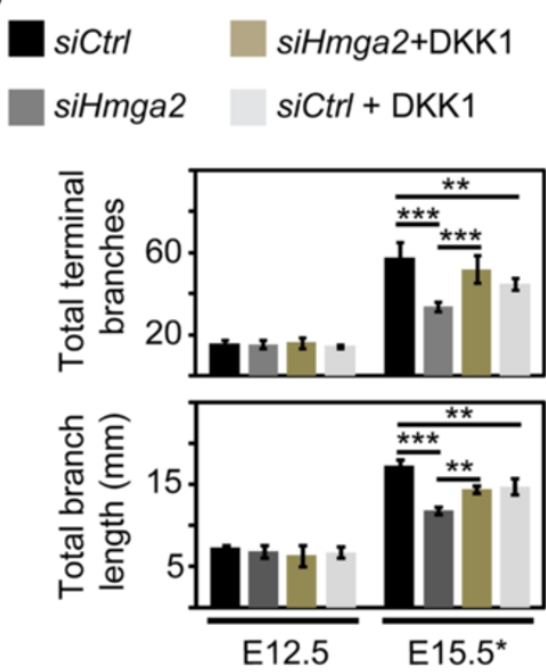

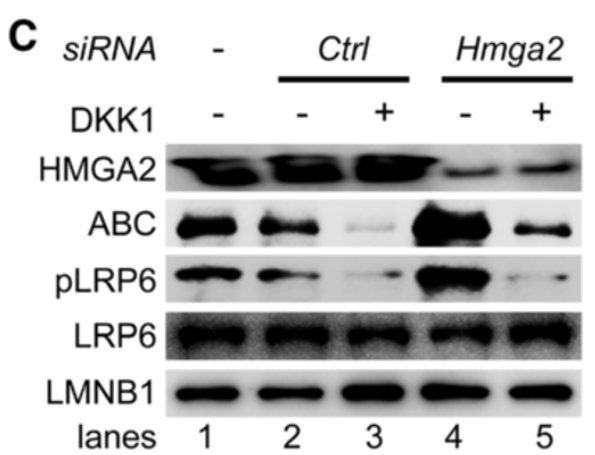

D
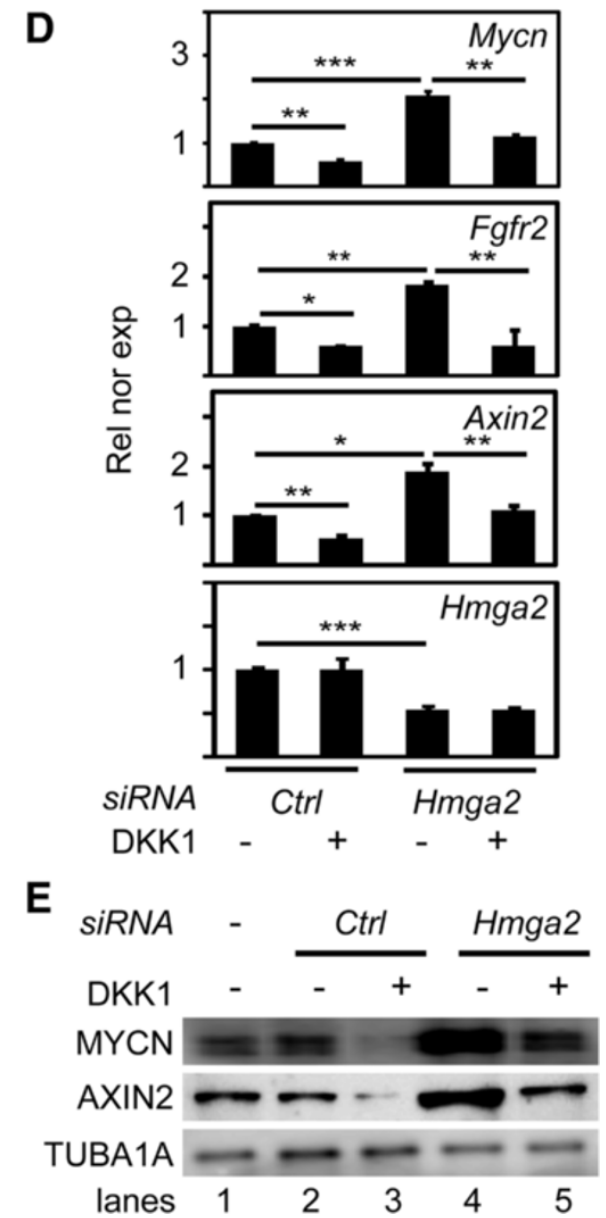

Figure 5 Inhibition of canonical WNT signaling partially rescued the Hmga2 loss-of-function phenotype. (A) Embryonic lung explants were cultured as in Additional file 4: Figure S4A. Explants were treated with control (siCtrl) or Hmga2-specific siRNAs (siHmga2) together with the canonical WNT inhibitor DKK1 for $72 \mathrm{~h}$ till stage E15 equivalent (E15*). Scale bars, $500 \mu \mathrm{m}$. (B) Terminal branches (top) and total branch length (bottom) were quantified at E12.5 and E15.5* Embryonic lung explants were treated as in A. Error bars show the SEM $(n=6)$. ${ }^{* * *} P<0.001$; ${ }^{*} P<0.01$; ${ }^{*} P<0.05$ after one-way analysis of variance. (C) Inhibition of canonical WNT signaling antagonized the effect of Hmga2-LOF. Protein extracts from the lung explants treated as in A were analyzed by western blot using the indicated antibodies. LMNB1 was used as loading control. (D) Expression analysis of the indicated genes by quantitative RT-PCR in embryonic lung explants that were treated as in A. Relative expression normalized to Tubala. Error bars show the SEM $(n=3)$. Asterisks as B. (E) Protein extracts from the lung explants treated as in A were analyzed by western blot using the indicated antibodies. TUBA1A was used as loading control.

terminal branches and branch length (Figure 5B). When E12.5 lungs were cultured for 72 hours in the presence of a Hmga2-specific siRNA (siHmga2), the total number of terminal branches was reduced from 58 ( $\mathrm{siCtrl}$ ) to 34
$(P<0.001 ; n=6)$, and the branch length from $17 \mathrm{~mm}$ (siCtrl) to $12 \mathrm{~mm}(P<0.001 ; n=6)$. However, treatment of the explants with the secreted canonical WNT inhibitor dickkopf homolog 1 (DKK1) [25] antagonized the 
defects caused by siHmga2 (Figure 5A,B), reconstituting the total number of terminal branches to $51(P<0.01$; $n=6)$ and the branch length to $14 \mathrm{~mm}(P<0.01 ; n=6)$. DKK1 treatment alone also affected branching morphogenesis by blocking WNT signaling as previously described from experiments in embryonic lung explants [26]. Western blot analysis of protein extracts from embryonic lung explants showed increased levels of $\mathrm{ABC}$ and phosphorylated LRP6 after Hmga2-KD (Figure 5C, lane 4), demonstrating enhanced canonical WNT signaling that was antagonized after DKK1 treatment (Figure 5C, lane 5). The partial rescue of the Hmga2LOF phenotype achieved by DKK1-mediated block of WNT signaling was validated by expression analysis of canonical WNT targets (Figure 5D) and by western blot analysis of protein extracts from embryonic lung explants (Figure 5E). Our data demonstrated the causal involvement of increased WNT signaling in mediating the effect of Hmga2-LOF, suggesting a role of Hmga2 in negative regulation of WNT signaling that was supported by reduced activity of the WNT-responsive p3LEF-LUC reporter after Hmga2 over-expression in MLE-12 cells (Additional file 3: Figure S3).

\section{HMGA2 directly activates Gata6 expression}

GATA6 is a transcription factor that is essential for branching morphogenesis and inhibits canonical WNT signaling in the distal epithelium of the lung by transcriptional activation of its downstream target, the canonical WNT-beta-catenin pathway antagonist Fzd2 [11,27]. The defects observed after Hmga2-LOF were similar to the Gata6-LOF phenotype (Additional file 2: Figure S2A,B) [9]. Therefore, we investigated whether the enhancement of canonical WNT signaling after Hmga2-LOF could involve Gata6 and Fzd2. Immunostaining on sections of embryonic lung (E14.5) using HMGA2- and GATA6-specific antibodies (Figure 6A) showed the presence of both proteins in the same cells of the distal lung endoderm. Interestingly, expression analysis in embryonic lung showed that Gata6- and Fzd2-transcripts decreased after Hmga2$\mathrm{KO}$ when compared to WT mice (Figure 6B). Consistently, siRNA-mediated Hmga2-LOF reduced Gata6 and Fzd2 expression in embryonic lung explants (Additional file 2: Figure S2B). Western blot analysis of protein extracts from embryonic lung (Figure 6C) showed reduced levels of GATA6 and FZD2 after Hmga2-KO, supporting our expression analysis. To investigate the effect of Hmga2
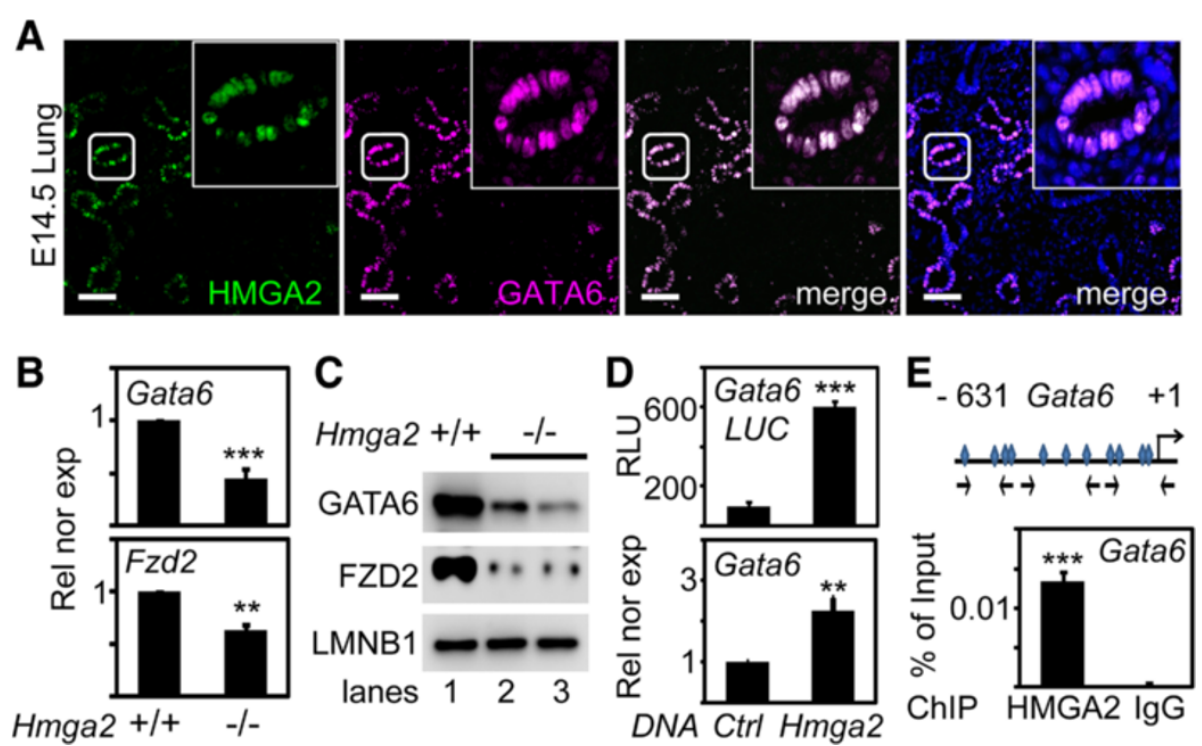

Figure 6 HMGA2 directly regulates Gata6 and thereby modulates Fzd2 expression. (A) HMGA2 and GATA6 co-localized in the nuclei of the same cells of the distal lung epithelium. Fluorescence microscopy of embryonic lung sections (E14.5) after double immunostaining using HMGA2-and GATA6-specific antibodies. Nuclear staining with DAPI (blue). Square shows details at higher magnification. Scale bars, $40 \mu \mathrm{m}$. (B) Hmga2-KO decreased expression of Gata6 and its downstream target gene Fzd2. Expression analysis of the indicated genes as in Figure 4D. Rel nor exp, relative expression normalized to Tuba 1a. Data are presented as mean \pm SEM $(n=4) .{ }^{* * *} P<0.001 ;{ }^{* *} P<0.01$ after one-way analysis of variance. (C) Protein extracts from WT (+/+) or Hmga2 ${ }^{-/-}$embryonic lung (E18.5) were analyzed as in Figure 4C using the indicated antibodies. (D) Hmga2 over-expression increased Gata6 transcription. Top, Luciferase reporter assays of MLE-12 cells transiently transfected with a Gata6-Luc reporter plasmid and control (Ctrl, empty vector) or Hmga2 expression construct. RLU, relative light units. Bottom, Gata6 expression was monitored by quantitative RT-PCR in MLE-12 cells treated as above. Rel nor exp, relative expression normalized to Gapdh. Data are presented as mean \pm SEM $(n=4)$ and asterisks as in B. (E) Endogenous HMGA2 binds to the endogenous Gata6 promoter. Top, in silico analysis of the Gata6 gene (-631 to +1 base pairs relative to transcription initiation site) revealed several HMGA2 binding elements (squares). Arrows, position of the primers used for ChIP. Bottom, ChIP of the Gata6 promoter using HMGA2-specific antibody or lgG (negative control). ChIP, chromatin-immunoprecipitation assay; IgG, immunoglobulin G. Data are presented as mean \pm SEM $(n=4)$ and asterisks as in B. 
gain-of-function (GOF) on Gata6 expression, MLE-12 cells were transiently transfected with $\mathrm{Hmga2}$ and a plasmid containing the luciferase $(L u c)$ reporter gene under the control of the Gata6 promoter (Figure 6D, top). Forced expression of Hmga2 increased more than fivefold the basal transcription of the Gata6-Luc reporter. In addition, expression of endogenous Gata6 increased more than twofold after Hmga2 transfection (Figure 6D, bottom). Consistently, in silico analysis of the murine Gata6 gene (Figure 6E, top) revealed several HMGA2 binding elements near the transcription initiation site. Moreover, chromatin immunoprecipitation assay (ChIP) in MLE-12 cells (Figure 6E, bottom) showed a direct association of endogenous HMGA2 to the endogenous Gata6 promoter. Thus, we conclude that HMGA2 directly activates Gata6 gene expression, and the enhanced WNT signaling caused by Hmga2-LOF could also be mediated by a reduction of the WNT signaling antagonizing proteins GATA6 and FZD2.

To show the requirement of Gata6 in Hmga2-mediated regulation of WNT signaling, we transfected MLE-12 cells with Hmga2 after siRNA-mediated Gata6- or Fzd2-depletion (Figure 7A). Expression analysis showed that Hmga2GOF increased the levels of Gata6 and $F z d 2$ but reduced the levels of canonical WNT targets Mycn, Axin 2 and Bmp4, confirming a role of Hmga2 in inhibition of canonical WNT signaling. Interestingly, Gata6- or Fzd2depletion antagonized the effect of Hmga2-GOF on both groups of markers analyzed, indicating the requirement of Gata6 and $F z d 2$ for the negative regulation of WNT signaling mediated by Hmga2.

To determine the causal involvement of Gata6 in Hmga2-mediated regulation of WNT signaling, we transfected MLE-12 cells with Gata6 after siRNA-mediated Hmga2- or Fzd2-depletion (Figure 7B and Additional file 3: Figure S3B). Expression analysis showed that Hmga2- and Fzd2-LOF enhanced the expression of canonical WNT targets, supporting our previous expression analysis in the embryonic lung of Hmga2-KO mice and as expected from WNT signaling antagonizing genes. Interestingly, Gata6 transfection compensated the effect of Hmga2-LOF, but not of $F z d 2$-LOF. Our data indicate that Gata 6 acts downstream of Hmga 2 and upstream of $F z d 2$ in negative regulation of WNT signaling (Figure 7C).

\section{Discussion and conclusions}

We showed that Hmga2 is expressed in the embryonic mouse lung at the distal airways. Hmga2 mRNA levels were high during early stages of lung development, in which cells are undifferentiated, and decreased as lung development progressed, coincident with cell differentiation. Interestingly, we detected a slight increase of Hmga2 expression at E18.5 that matches with the establishment of a bipotent progenitor cell population in the distal epithelium [28]. Our data correlate with previous reports where HMGA2 was shown to be present at high levels in various undifferentiated tissues during embryonic development and in strongly reduced levels in the corresponding adult tissues [12-15].

Hmga2-KO induces a pygmy phenotype due to reduced expression of insulin-like growth factor 2 mRNA binding protein 2 (Igf2bp2) [15,16,29]. Prior to our study, the lung of $\mathrm{Hmga2}^{-/-}$mice had not been analyzed. Detailed analysis of $\mathrm{Hmga2}^{-/-}$mice showed that Hmga2 is required for distal epithelium differentiation during embryonic lung development. Hmga2-KO led to enhanced canonical WNT signaling due to an increase of secreted WNT glycoproteins as well as a reduction of the WNT signaling antagonizing proteins GATA6 and FZD2, thereby supporting that Hmga 2 regulates WNT signaling at different points of the pathway (Figure 7C). The causal involvement of canonical WNT signaling in mediating the effect of Hmga2-LOF was demonstrated by the DKK1-induced rescue of Hmga2-LOF in embryonic lung explants (Figure 5A-E). HMGA2-mediated regulation of Gata6 seems to be a key process in fine-tuning the activity of canonical WNT signaling in airway epithelium. The sequential order of events suggested in our model (Figure 7C) in which Hmga2 acts upstream of Gata6 is strongly supported by the fact that HMGA2 directly regulates Gata6 (Figure 6A-E) as well as by the Gata6mediated rescue experiments of Hmga2-LOF in MLE-12 cells (Figure 7B). Since HMGA2 regulates the transcription of its target genes by modulating the chromatin structure and by recruiting other proteins to the transcription regulatory complex [12], the scope of our future work will be to investigate in detail the mechanism of HMGA2-mediated transcriptional regulation of the Gata6 promoter.

Hmga2-KO increased cell proliferation not only in the lung epithelium, where Hmga2 is expressed, but also in the mesenchyme, suggesting a paracrine effect that could be explained by increased expression of the secreted components of WNT signaling. Since HMGA2 is known to activate transcription, the increased expression of Wnt2b, Wnt7b and Wnt11 after Hmga2-KO suggest the participation of a transcription inhibitor that could block the expression of these secreted components of WNT signaling and whose expression could be regulated by HMGA2 (Figure 7C). Identification of this unknown mediator of HMGA2 will be the scope of future studies. Interestingly, analysis of the $W n t 7 b$ promoter showed that deletion of the region between -1,005 bp and -829 bp relative to the second transcription start site significantly increased the basal transcription activity of a $W n t 7 b$-luciferase reporter [30], suggesting that the binding element of a putative transcription inhibitor was deleted in this construct. 


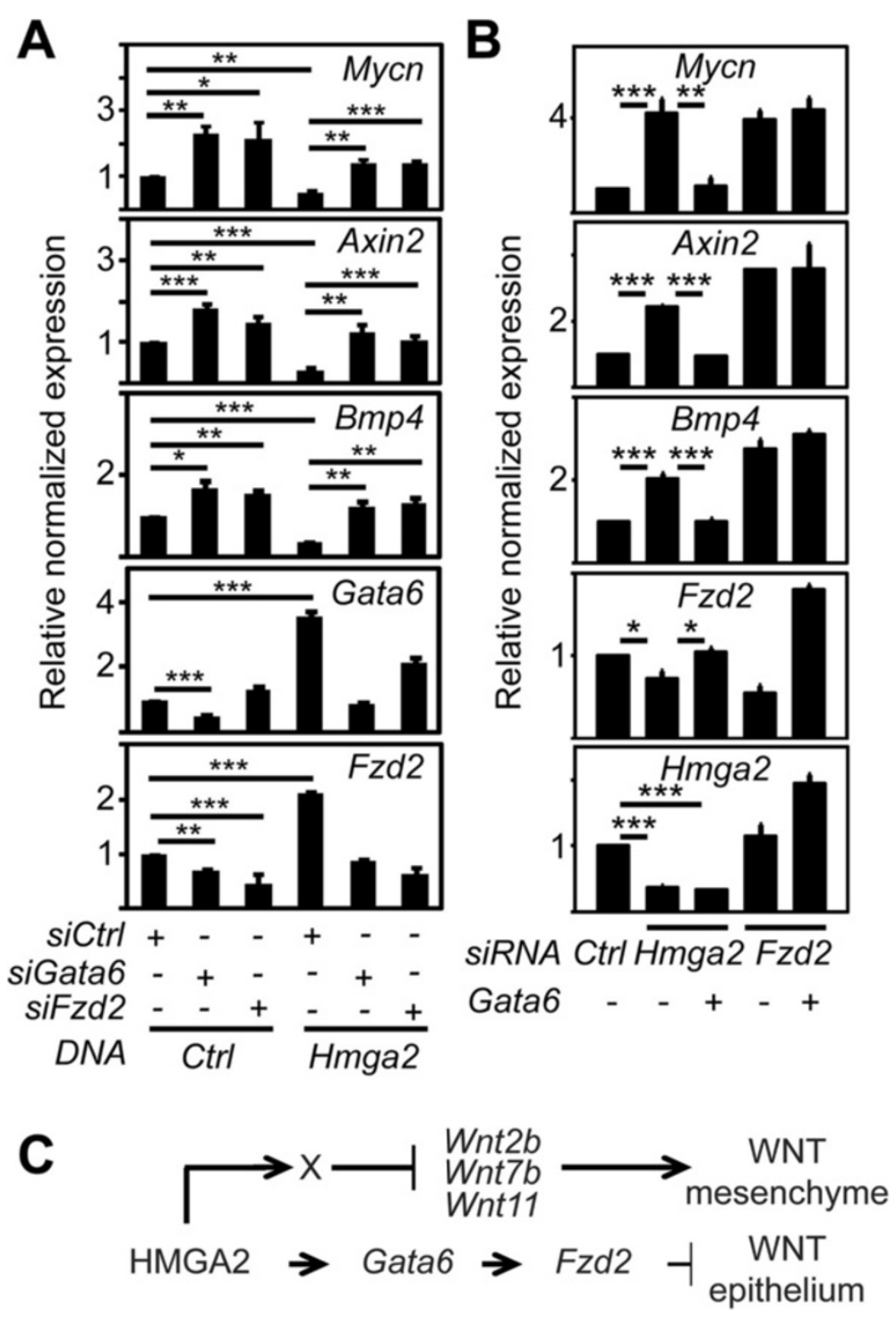

Figure 7 Hmga2 acts upstream of Gata6 in WNT signaling regulation. (A) Gata6 and Fzd2 are required for the effect of Hmga2 GOF on expression of WNT targets. Expression analysis of the indicated genes by quantitative RT-PCR in MLE-12 cells that were transfected with either control (Ctrl) or Gata6- or Fzd2-specific siRNA and Hmga2 expression plasmid as indicated. Data are presented as mean \pm SEM $(n=4)$. ${ }^{* * *} P<0.001$; ${ }^{* * P}<0.01$; ${ }^{*} P<0.05$ after one-way analysis of variance. (B) Gata6-GOF rescued the effect of Hmga2-LOF on expression of WNT targets. Expression analysis of the indicated genes by quantitative RT-PCR in MLE-12 cells that were transfected with either control (Ctr) or Hmga2- or Fzd2-specific siRNA and Gata6 expression plasmid as indicated. Data are presented as mean \pm SEM $(n=4)$ and asterisks as in A. (C) Model. Hmga2 regulates WNT signaling at different points of the pathway. Regulation of the secreted WNT glycoproteins (Wnt2b, Wnt7b and Wnt11) mediates the paracrine effect on the mesenchyme of the embryonic lung. In addition, HMGA2-mediated regulation of Gata6 is a key process in fine-tuning the activity of canonical WNT signaling in developing airway epithelium. $X$, unknown transcription factor.

The phenotypes of Hmga2- and Gata6-LOF in embryonic lung explants are very similar (Additional file 2: Figure S2A,B) [9]. However, the milder phenotype observed in the embryonic lung of Hmga2-KO mice when compared either with the Gata6-KO or the phenotype induced after Hmga2-KD in embryonic lung explants might be explained by redundancy in the function between Hmga 2 and Hmgal, another member of the HMG protein family. Hmga1 transcript was reduced after Hmga2-LOF
(Additional file 4: Figure S4C) but not affected in the Hmga2-KO mice (Additional file 1: Figure S1B). Hmga1 might compensate the Hmga2-KO, thereby avoiding lethality at early embryonic stages, as is the case after Gata6-KO [31,32], or soon after birth due to defects in the lung, as is the case after lung epithelium-specific ablation of Gata6 [11]. In addition, the expansion of the mesenchyme in the embryonic lung after Hmga2-KO and the apparent increase of epithelium in embryonic lung 
explants after Hmga2-KD might be explained by the differences of both LOF systems. In the transgenic approach, Hmga2-LOF takes place soon after fertilization and affects lung development from the initial stages of lung bud formation; in the explant culture, the LOF starts at E12.5, thereby reducing the rather indirect effect on the mesenchyme and making the effect on the epithelium more dominant. Analysis of the lungs in inducible and conditional double transgenic mice $\left(\mathrm{Hmga2}^{-/-}: \mathrm{Hmga1}^{-/-}\right)$would test these hypotheses and should be the scope of future studies.

Hmga 2 expression is positively regulated by transforming growth factor beta 1 signaling [33]. In addition, our data show that Hmga2 antagonizes canonical WNT signaling. Therefore, it will be of interest to determine a potential opposing effect between these two signaling pathways in establishing the proximal-distal axis during branching morphogenesis and lung epithelium differentiation in the developing lung. Hmga2 might play a crucial role on the interplay between these signaling pathways.

Organ regeneration requires a proper balance between self-renewal and differentiation of tissue-specific progenitor cells. Canonical WNT signaling has been implicated in different regenerative processes including zebrafish tail regeneration, zebrafish cardiac regeneration and expansion of anterior heart field progenitors in mammals [34,35]. Moreover, canonical WNT signaling is activated upon lung epithelial regeneration, and enhanced WNT activity caused by lung epithelium-specific ablation of Gata6 led to a premature and increased number of BASCs [11]. BASCs represent one of several regional progenitor cell populations in the adult lung and are responsible for regeneration of bronchiolar and alveolar epithelium during homeostatic turnover and in response to injury $[4,36]$. Our study suggests the possible role of Hmga2 in the adult lung controlling the balance between BASC expansion and differentiation. Our results are the starting point for future studies in which the relevance of Hmga2-mediated regulation of WNT signaling might be investigated in the adult lung within the context of proper balance between differentiation and self-renewal of lung stem/progenitor cells and lung regeneration during both homeostatic turnover and repair after injury. Characterization of the regulatory mechanisms controlling the proper balance between expansion and differentiation of lung stem/progenitor cells will have a profound impact on our understanding and treatment of lung disease.

\section{Methods}

\section{Animal experiments}

Mouse work was performed in compliance with the German Law for Welfare of Laboratory Animals. The permission to perform the experiments presented in this study was obtained from the Regional Council (Regierungspräsidium in Darmstadt, Germany). The numbers of the permissions are IVMr46-53r30.03. MPP04.12.02 and IVMr46-53r30.03.MPP06.12.01. Animals were killed for scientific purposes according to the law mentioned above, which complies with national and international regulations.

C57BL/6 and $\mathrm{Hmga2}^{+/-}$mice (stock \# 002644, Jackson Laboratories) [37] were obtained from Charles River Laboratories (Germany) at 5 to 6 week of age. BAT-GAL transgenic reporter mice (also called beta-catenin/TCF/ LEF reporter transgenic mice) were obtained as a gift from Prof. Stefan Liebner [38]. BAT-GAL:Hmga2 ${ }^{-/-}$double transgenic embryos were obtained by crossing heterozygous BAT-GAL:Hmga2 ${ }^{+-}$mice. Animals were housed and bred under controlled temperature and lighting (12/ 12-hour light/dark cycle), fed with commercial animal feed and water ad libitum. All experiments were performed with mouse embryonic lungs. Timed-pregnant C57BL/6 WT mice were killed at indicated time points; embryonic lungs were isolated according to standard methods and whole-mount in situ hybridization was performed as described [39] with minor modifications. Briefly, to synthesize digoxigenin-labeled RNA probes, pcDNA3-mHmga2 plasmid (gift from Prof. Peter Grouse) [40] was linearized, and UTP-digoxigenin (Roche) substituted antisense RNA probes were transcribed with T7 RNA polymerase. Sense RNA probes as negative control were transcribed with SP6 RNA polymerase.

\section{Embryonic lung explants culture}

Lungs of timed-pregnant C57BL/6 WT mice were dissected from the embryos at E12.5 and cultured for 72 hours till E15.5 equivalent (E15.5*) as previously reported [24]. The explants were treated with $3 \mu \mathrm{M}$ siRNAs against Hmga2 (Applied Biosystems, Silencer Select siRNAs, Assay ID s67600), scrambled siRNA (negative control, Ctrl) (Sigma, MISSION siRNA Universal Negative Control, SIC001) or $200 \mathrm{nM}$ of mouse recombinant DKK1 (R\&D Systems, 5897-DK-010) following a similar protocol as previously described [9,41-44]. The siRNA and protein treatments were renewed every 24 hours. After 72 hours, the lungs were checked for morphological changes by standard microscopy techniques and harvested for RNA (QIAGEN RNeasy Micro Kit) and protein isolation. The images were used to determine the total number of terminal bud branches and for quantification of total branch length as described [45].

\section{Cell culture transfection assays}

Mouse lung epithelial cells (MLE-12, ATCC CRL-2110) were cultured following the supplier's instructions. MLE-12 cells were transiently transfected either with $40 \mathrm{nM}$ siCtrl (negative control; AM4611, Ambion), $40 \mathrm{nM}$ siGata6 (L-065585-00, Dharmacon), $20 \mathrm{nM} \mathrm{siFzd2} \mathrm{(s81164,} \mathrm{Applied}$ Biosystems), 20 nM siHmga2 (s67600, Applied 
Biosystems) and/or pcDNA 3.1(A)-Hmga2-myc/His or $p C M V 6$-entry-Gata6-flag/myc or $p B L$ (Ctrl) as indicated using Lipofectamine 2000 transfection reagent (Invitrogen) at a ratio of 1:2 DNA:Lipofectamine. Cells were harvested 48 hours later for further analysis.

The proximal 631 bp Gata6 promoter was amplified and cloned into the pGL4basic vector to generate $p G L 4$ Gata6 promoter luciferase vector. Dual-luciferase reporter assays (Promega) were performed as described [46] following transient transfection of MLE-12 cells in 96-well plates with $20 \mathrm{nM}$ effector siRNAs and a total of $100 \mathrm{ng}$ DNA per well, containing $15 \mathrm{ng}$ effector plasmid, $15 \mathrm{ng}$ $p$ GL4-Gata6 promoter or p3LEF-LUC luciferase reporter plasmid, 1 ng Renilla luciferase reporter plasmid and 69 ng pBluescript. Each sample was performed in triplicate. Each experiment was repeated at least three times.

\section{Affymetrix microarrays, quantitative PCR and ChIP assays} Total RNA was isolated with RNeasy plus mini kit (Qiagen) and quantified using a spectrophotometer. Affymetrix microarray-based transcriptome analysis of $\mathrm{Hmga2}^{-/-}$and $\mathrm{Hmga2}^{+/+}$embryonic lung (E18.5) was performed and analyzed as described [47]. Kyoto Encyclopedia of Genes and Genomes pathway enrichment based analysis of dysregulated pathways in $\mathrm{Hmga2}^{-/-}$versus $\mathrm{Hmga2}^{+/+}$was done using DAVID software [48] and generation of fold change and Heat map were performed using DNAStar Arraystar 11.0. The data discussed in this publication have been deposited in NCBI's Gene Expression Omnibus [49] through accession number [GEO:GSE55340] (http://www. ncbi.nlm.nih.gov/geo/query/acc.cgi?acc=GSE55340).

The High Capacity Reverse Transcription kit (Applied Biosystems) was used for synthesis of cDNA from total RNA. Quantitative real-time PCR reactions were performed using SYBR ${ }^{\circ}$ Green on the Step One plus Realtime PCR system (Applied Biosystems). The PCR results were normalized with respect to the housekeeping gene for tubulin alpha 1a (Tuba1a) or glyceraldehyde-3-phosphate dehydrogenase (Gapdh).

ChIP analysis of the mouse Gata6 promoter was performed as described [50] with slight modifications. Briefly, MLE-12 cells were cross-linked by $1 \%$ formaldehyde for 10 minutes, lysed, and sonicated with Diagenode Biorupter to an average DNA length of 500 to $600 \mathrm{bp}$. After isolation, the soluble chromatin was immunoprecipitated with immunoglobulin G (control, Santa Cruz) or HMGA2-specific antibody (sc-30223; Santa Cruz Biotechnology). Reverse cross-linked immunoprecipitated chromatin was subjected to qPCR using the primers listed in Additional file 5: Table S1.

\section{Immunohistochemistry}

For paraffin-embedded mouse embryonic lung tissue, lungs were fixed overnight in $1 \%$ paraformaldehyde at $4^{\circ} \mathrm{C}$, dehydrated over a graded series of alcohol, and paraffin embedded. Sections of $4 \mu \mathrm{m}$ were prepared on a microtome (Leica). Antigen retrieval was performed by microwave heating for 8 minutes using $1 \mathrm{mM}$ EDTA (pH 8) or $1 \mathrm{mM}$ citrate buffer (pH 6). For cryosections of mouse lung tissue, lungs were harvested and embedded in polyfreeze tissue freezing medium (Polysciences). Sections of $10 \mu \mathrm{m}$ were prepared on a cryostat (Leica). Sections were post-fixed in $4 \%$ paraformaldehyde for 10 minutes. Antibody staining was performed following standard procedures. All incubations and washes were performed with histobuffer containing $3 \%$ bovine serum albumin and $0.2 \%$ Triton X-100 in $1 \times$ phosphate-buffered saline, pH 7.4. Non-specific binding was blocked by incubating with donkey serum and histobuffer $(1: 1(\mathrm{v} / \mathrm{v})$ ratio) for 45 to 60 minutes. The sections were then incubated with primary and secondary antibodies for 60 minutes followed by nuclear staining. The sections were examined with a confocal microscope (Zeiss) or fluorescent microscope (Leica). Antibodies used were specific against HMGA2 (BioCheck), LMNB1 (Santa Cruz), GATA6 (R\&D system), cadherin 1 (Abcam), VIM-Cy3 (Sigma), PCNA (Santa Cruz), MKi67 (Abcam), KRT (Dako and Sigma), ProSFTPC (Millipore), SOX9 (Santa Cruz), ABC (Millipore), CTNNB1 (Abcam), ACTA2-Cy3 (Sigma) and clCASP3 (Cell Signaling). Secondary antibodies used were Alexa 488, Alexa 633 and Alexa 555 (Invitrogen). DAPI (Invitrogen) were used as nuclear dye.

Paraformaldehyde-fixed and paraffin-embedded lung tissue sections were stained with hematoxylin and eosin and used for the lung morphology analysis. Figures were elaborated following a color scheme recommended to make them visible and easy to interpret by people with all types of color vision [51,52].

\section{Western blot}

Western blotting was performed following standard protocols and using antibodies specific for HMGA2 (Biocheck and Santa Cruz), TUBA1A (Sigma), GATA6 (R\&D system), FZD2 (Abcam), active-beta-catenin (ABC, Millipore), LRP6 (Cell signaling), phosphorylated LRP6 (Cell signaling), AXIN2 (Abcam), BMP4 (Millipore), MYCN (Santa Cruz) and LMNB1 (Santa Cruz). Immunoreactive proteins were visualized with the corresponding horseradish peroxide-conjugated secondary antibodies using the Super Signal West Femto detection solutions (Thermo Scientific). Signals were detected and analyzed with Luminescent Image Analyzer (Las 4000, Fujifilm).

\section{Statistical analysis}

In total, the lungs of six $H m g a 2^{-/-}$mice were analyzed using different techniques. The lungs of six $\mathrm{Hmga2}^{+/+}$ were used as control because the heterozygote $\mathrm{Hmga2}^{+/-}$ mice presented a mild phenotype. With the exception of 
the Affymetrix array-based expression analysis, the experiments were performed at least three times and the samples in each experiment were analyzed in triplicates. The Affymetrix array-based expression analysis was performed one time using biological duplicates. Statistical analyses were performed using Excel Solver. All data are represented as mean \pm SEM. One-way analyses of variance (ANOVA) were used to determine the levels of difference between the groups and $P$ values for significance.

\section{Additional files}

Additional file 1: Figure S1. Characterization of embryonic lung in $\mathrm{Hmga2}^{-/-}$mice. (A) Top, $\mathrm{Hmga2}^{-/-}$mouse embryos at E18.5 were smaller than WT embryos. Main interval of the scale is equivalent to $1 \mathrm{~cm}$. Bottom, $\mathrm{Hmga2}^{-/-}$embryo showed $40 \%$ body wet weight reduction when compared to WT embryos. Data are represented as mean \pm SEM $(n=3)$. ${ }^{* * *} P<0.001$; ${ }^{* *} P<0.01 ;{ }^{*} P<0.05$. (B) Expression analysis of the indicated genes by qRT-PCR in embryonic lung (E18.5) of WT and $\mathrm{Hmga2}^{-/-}$mice. Rel nor exp, relative expression normalized to Tubala.; Scgb1a1, secretoglobin 1A1 also known as CC10; Sftpc, surfactant-associated protein $C$ also known as SPC. Error bars, SEM $(n=4)$. Asterisks as in A. (C) Sections of embryonic lung (E18.5) of WT (+/+) and $\mathrm{Hmga2}^{-1-}$ mice were analyzed by confocal microscopy after double immunostaining using (left) MKI67- and $\mathrm{CDH} 1$-specific antibodies or (right) PCNA- and VIM-specific antibodies. Nuclear staining with DAPI (blue). Scale bars, $40 \mu \mathrm{m}$.

Additional file 2: Figure S2. Hmga2 and Gata6 loss-of-function in embryonic lung explants led to a marked impairment of airway epithelial differentiation due to enhanced canonical WNT activity. (A) Hmga2-KD in lung explants led to dilated airways and resulted in lung epithelial differentiation defects. Phenotype of Gata6-KD in lung explants was similar to the Hmga2-KD. Top, embryonic lungs were explanted and cultured until E15.5* as in Additional file 4: Figure S4A. Explants were treated with control (siCtr), Hmga2- (siHmga2) or Gata6- (siGata6) specific siRNAs. Scale bars, $500 \mu \mathrm{m}$. Bottom, sections of treated explants were analyzed by confocal microscopy after immunostaining using either SFTPC- or with ABC-specific antibodies as indicated. Nuclear staining with DAPI (blue). ABC, activated-beta-catenin. Scale bars, $40 \mu \mathrm{m}$. (B) Hmga2 or Gata6 knockdown enhanced expression of canonical WNT pathway markers and reduced Fzd2 expression. Expression analysis of the indicated genes as in Additional file 4: Figure S4C. Gene expression normalized to Tubla1. Fzd2, frizzled homolog 2; Fgfr2, fibroblast growth factor receptor 2 . Error bars, SEM $(n=4)$. ${ }^{* *} P<0.001$; ${ }^{*} P<0.01 ;{ }^{*} P<0.05$.

Additional file 3: Figure S3. Hmga2 depletion increased the transcription of a WNT-responsive reporter whereas Hmga2 forced expression reduced it. (A) Luciferase reporter assays of MLE-12 cells transiently transfected with a p3LEF-LUC reporter plasmid and control (Ctrl) or Hmga2 specific siRNA (siHmga2); or Hmga2 expression construct; or treated with lithium chloride (LiCl, positive control). Error bars, SEM $(n=3)$. (B) Gata6-GOF rescued the effect of Hmga2-LOF on expression of WNT targets. Expression analysis of the indicated genes by qRT-PCR in MLE-12 cells that were transfected with either control (Ctrl) or Hmga2- or Fzd2-specific siRNA and Gata6 expression plasmid as indicated. Data are represented as mean \pm SEM $(n=4)$. ${ }^{* *} P<0.001 ;{ }^{*} P<0.01 ;{ }^{*} P<0.05$.

Additional file 4: Figure S4. Hmga2 loss-of-function in embryonic lung explants led to a marked impairment of airway epithelial differentiation due to enhanced canonical WNT activity confirming the results obtained in $\mathrm{Hmga2}^{-/-}$mice. (A) Schematic diagram of embryonic lung explant culture experiments. Mice embryonic lungs were explanted at E12.5, cultured for 72 hours until E15.5* and harvested for gene expression analysis and immunostaining. Scale bars, $500 \mu \mathrm{m}$. (B) Embryonic lung explant cultures mimic the normal embryonic lung development. Expression analysis of the indicated genes by qRT-PCR in embryonic lungs (E12.5, E15.5) and in explanted lungs (E15.5*) as in A. Rel nor exp, relative expression normalized to Tubla1. Data are represented as mean $\pm \operatorname{SEM}(n=4)$. (C) SiRNA-mediated Hmga2-LOF was efficient in lung explants. Embryonic lungs were explanted and cultured until E15.5* as in A. Explants were treated with control (siCtrl) or Hmga2-specific (siHmga2) siRNAs. Expression of the indicated genes was analyzed by qRT-PCR. Rel nor exp, relative expression normalized to Tub1a1. Error bars, SEM $(n=4)$. ${ }^{* * *} P<0.001 ;{ }^{*} P<0.05$. (D) Lung explants were treated with siRNAs as in C. Protein extracts of treated explants were analyzed by western blot using HMGA2-, ABC- or TUBA1A-specific antibodies. (E) Hmga2-KD enhanced the activity of the beta-catenin/TCF/LEF reporter in lung explants of the BAT-GAL transgenic mice. Lung explants of BAT-GAL mice were cultured as in A. Explants were treated with control (siCtr) or Hmga2-specific siRNAs (siHmga2) or with lithium chloride (LiCl, positive control). Beta-galactosidase staining was performed with the treated explants to detect activated WNT signaling (blue color). Scale bars, $500 \mu \mathrm{m}$.

Additional file 5: Table S1. Oligonucleotide sequences used for real-time qPCR and ChIP.

\section{Abbreviations}

BASC: bronchioalveolar stem cells; bp: base pairs; ChIP: chromatin imunoprecipitation; GOF: gain of function; KD: knockdown; KO: knockout; LOF: loss of function; QRT-PCR: quantitative reverse transcription polymerase chain reaction; siRNA: short interfering RNA; WT: wild type; E15.5*: E15.5 equivalent.

\section{Competing interests}

The authors declare that they have no competing interests.

\section{Authors' contributions}

Luc-assays, western blots, in situ hybridization and ChIPs were performed by IS; immunohistochemistry by IS and AM; RT-PCR by IS, GB, AM and AC; embryonic lung explants culture by AM, IS, MW and GC; Affymetrix microarray-based expression analysis by $\mathrm{TB}$ and $\mathrm{AC} ; \mathrm{TB}, \mathrm{MW}, \mathrm{HACF}, \mathrm{TBr}, \mathrm{WS}, \mathrm{SB}$ and $\mathrm{GC}$ were involved in study design; GB and IS designed the study, analyzed data and wrote the manuscript. All authors discussed the results and commented on the manuscript. All authors read and approved the final manuscript.

\section{Authors' information}

All affiliations in Germany are members of the Universities of Giessen and Marburg Lung Center (UGMLC) and the German Center of Lung Research (DZL).

\section{Acknowledgments}

We thank R Bender and K Goth for technical support; S Liebner, P Grouse, PR Strauss, J Kwon for reagents and VS Nikam, R Voswinckel and N Oeztuerk for helpful discussions. This work was done according to the program of competitive growth of the Kazan Federal University and the Russian Government. GB is funded by the "LOEWE-Initiative der Landesförderung" (Wiesbaden Germany) (III L 4 - 518/15.004 2009) and the "Deutsche Forschungsgemeinschaft" (DFG, Bonn, Germany) (BA 4036/1-1).

\section{Author details}

'LOEWE Research Group Lung Cancer Epigenetic, Max-Planck-Institute for Heart and Lung Research, Parkstraße 1, 61231 Bad Nauheim, Germany. ${ }^{2}$ Department of Cardiac Development and Remodeling, Max-Planck-Institute for Heart and Lung Research, Parkstraße 1, 61231 Bad Nauheim, Germany. ${ }^{3}$ Chair for Lung Matrix Remodeling, Excellence Cluster Cardio Pulmonary System, Justus-Liebig- University, 35932 Giessen, Germany. ${ }^{4}$ Biochemistry Institute, Medical School, Justus-Liebig- University, 35932 Giessen, Germany. ${ }^{5}$ Institute of Fundamental Medicine and Biology, Kazan (Volga Region) Federal University, 18 Kremlyovskaya Street, Kazan 420008, Russian Federation. ${ }^{6}$ Department of Lung Development and Remodeling, Max-Planck-Institute for Heart and Lung Research, Parkstraße 1, 61231 Bad Nauheim, Germany.

Received: 27 December 2013 Accepted: 10 March 2014 Published: 24 March 2014

\section{References}

1. Costa RH, Kalinichenko W, Lim L: Transcription factors in mouse lung development and function. Am J Physiol Lung Cell Mol Physiol 2001, 280:L823-L838. 
2. Warburton D, Schwarz M, Tefft D, Flores-Delgado G, Anderson KD, Cardoso WV: The molecular basis of lung morphogenesis. Mech Dev 2000, 92:55-81.

3. Cardoso WV, Lu J: Regulation of early lung morphogenesis: questions, facts and controversies. Development 2006, 133:1611-1624.

4. Kim CF, Jackson EL, Woolfenden AE, Lawrence S, Babar I, Vogel S, Crowley D, Bronson RT, Jacks T: Identification of bronchioalveolar stem cells in normal lung and lung cancer. Cell 2005, 121:823-835.

5. Bellusci S, Grindley J, Emoto H, Itoh N, Hogan BL: Fibroblast growth factor 10 (FGF10) and branching morphogenesis in the embryonic mouse lung. Development 1997, 124:4867-4878.

6. Weaver M, Yingling JM, Dunn NR, Bellusci S, Hogan BL: Bmp signaling regulates proximal-distal differentiation of endoderm in mouse lung development. Development 1999, 126:4005-4015.

7. Hogan BL: Morphogenesis. Cell 1999, 96:225-233.

8. Morrisey EE, Ip HS, Lu MM, Parmacek MS: GATA-6: a zinc finger transcription factor that is expressed in multiple cell lineages derived from lateral mesoderm. Dev Biol 1996, 177:309-322.

9. Keijzer R, van Tuyl M, Meijers C, Post M, Tibboel D, Grosveld F, Koutsourakis $M$ : The transcription factor GATA6 is essential for branching morphogenesis and epithelial cell differentiation during fetal pulmonary development. Development 2001, 128:503-511.

10. Yang $H$, Lu MM, Zhang L, Whitsett JA, Morrisey EE: GATA6 regulates differentiation of distal lung epithelium. Development 2002, 129:2233-2246.

11. Zhang Y, Goss AM, Cohen ED, Kadzik R, Lepore JJ, Muthukumaraswamy K, Yang J, DeMayo FJ, Whitsett JA, Parmacek MS, Morrisey EE: A Gata6-Wnt pathway required for epithelial stem cell development and airway regeneration. Nat Genet 2008, 40:862-870.

12. Pfannkuche K, Summer H, Li O, Hescheler J, Droge P: The high mobility group protein HMGA2: a co-regulator of chromatin structure and pluripotency in stem cells? Stem Cell Rev 2009, 5:224-230.

13. Ashar HR, Chouinard RA Jr, Dokur M, Chada K: In vivo modulation of HMGA2 expression. Biochim Biophys Acta 2010, 1799:55-61.

14. Nishino J, Kim I, Chada K, Morrison SJ: Hmga2 promotes neural stem cell self-renewal in young but not old mice by reducing p16Ink4a and p19Arf expression. Cell 2008, 135:227-239.

15. Li Z, Gilbert JA, Zhang Y, Zhang M, Qiu Q, Ramanujan K, Shavlakadze T, Eash JK, Scaramozza A, Goddeeris MM, Kirsch DG, Campbell KP, Brack AS, Glass DJ: An HMGA2-IGF2BP2 axis regulates myoblast proliferation and myogenesis. Dev Cell 2012, 23:1176-1188.

16. Zhou X, Benson KF, Ashar HR, Chada K: Mutation responsible for the mouse pygmy phenotype in the developmentally regulated factor HMGI-C. Nature 1995, 376:771-774

17. Del Riccio V, van Tuyl M, Post M: Apoptosis in lung development and neonatal lung injury. Pediatr Res 2004, 55:183-189.

18. Zeng X, Tamai K, Doble B, Li S, Huang H, Habas R, Okamura H, Woodgett J, He X: A dual-kinase mechanism for Wnt co-receptor phosphorylation and activation. Nature 2005, 438:873-877.

19. Mao B, Wu W, Li Y, Hoppe D, Stannek P, Glinka A, Niehrs C: LDL-receptorrelated protein 6 is a receptor for dickkopf proteins. Nature 2001, 411:321-325.

20. Bilic J, Huang YL, Davidson G, Zimmermann T, Cruciat CM, Bienz M, Niehrs C: Wnt induces LRP6 signalosomes and promotes dishevelleddependent LRP6 phosphorylation. Science 2007, 316:1619-1622.

21. Reya T, Duncan AW, Ailles L, Domen J, Scherer DC, Willert K, Hintz L, Nusse R, Weissman IL: A role for Wnt signalling in self-renewal of haematopoietic stem cells. Nature 2003, 423:409-414.

22. Pei $Y$, Brun SN, Markant SL, Lento W, Gibson P, Taketo MM, Giovannini M, Gilbertson RJ, Wechsler-Reya RJ: WNT signaling increases proliferation and impairs differentiation of stem cells in the developing cerebellum. Development 2012, 139:1724-1733.

23. Rulifson IC, Karnik SK, Heiser PW, ten Berge D, Chen H, Gu X, Taketo MM, Nusse R, Hebrok M, Kim SK: Wnt signaling regulates pancreatic beta cell proliferation. Proc Natl Acad Sci USA 2007, 104:6247-6252.

24. Del Moral PM, Warburton D: Explant culture of mouse embryonic whole lung, isolated epithelium, or mesenchyme under chemically defined conditions as a system to evaluate the molecular mechanism of branching morphogenesis and cellular differentiation. Methods Mol Biol 2010, 633:71-79
25. Kazanskaya O, Glinka A, Niehrs C: The role of Xenopus dickkopf1 in prechordal plate specification and neural patterning. Development 2000, 127:4981-4992.

26. De Langhe SP, Sala FG, Del Moral PM, Fairbanks TJ, Yamada KM, Warburton D, Burns RC, Bellusci S: Dickkopf-1 (DKK1) reveals that fibronectin is a major target of Wnt signaling in branching morphogenesis of the mouse embryonic lung. Dev Biol 2005, 277:316-331.

27. Ishitani T, Kishida S, Hyodo-Miura J, Ueno N, Yasuda J, Waterman M, Shibuya H, Moon RT, Ninomiya-Tsuji J, Matsumoto K: The TAK1-NLK mitogen-activated protein kinase cascade functions in the Wnt-5a/Ca (2+) pathway to antagonize Wnt/beta-catenin signaling. Mol Cell Biol 2003, 23:131-139.

28. Desai TJ, Brownfield DG, Krasnow MA: Alveolar progenitor and stem cells in lung development, renewal and cancer. Nature 2014, 507:190-194.

29. Brants JR, Ayoubi TA, Chada K, Marchal K, van de Ven WJ, Petit MM: Differential regulation of the insulin-like growth factor II mRNA-binding protein genes by architectural transcription factor HMGA2. FEBS Lett 2004, 569:277-283.

30. Weidenfeld J, Shu W, Zhang L, Millar SE, Morrisey EE: The WNT7b promoter is regulated by TTF-1, GATA6, and Foxa2 in lung epithelium. J Biol Chem 2002, 277:21061-21070.

31. Koutsourakis M, Langeveld A, Patient R, Beddington R, Grosveld F: The transcription factor GATA6 is essential for early extraembryonic development. Development 1999, 126:723-732.

32. Morrisey EE, Tang Z, Sigrist K, Lu MM, Jiang F, Ip HS, Parmacek MS: GATA6 regulates HNF4 and is required for differentiation of visceral endoderm in the mouse embryo. Genes Dev 1998, 12:3579-3590.

33. Thuault S, Valcourt U, Petersen M, Manfioletti G, Heldin CH, Moustakas A: Transforming growth factor-beta employs HMGA2 to elicit epithelialmesenchymal transition. J Cell Biol 2006, 174:175-183.

34. Stoick-Cooper CL, Weidinger G, Riehle KJ, Hubbert C, Major MB, Fausto N, Moon RT: Distinct Wnt signaling pathways have opposing roles in appendage regeneration. Development 2007, 134:479-489.

35. Cohen ED, Wang Z, Lepore JJ, Lu MM, Taketo MM, Epstein DJ, Morrisey EE: Wnt/beta-catenin signaling promotes expansion of |s|-1-positive cardiac progenitor cells through regulation of FGF signaling. J Clin Invest 2007, 117:1794-1804.

36. Giangreco A, Reynolds SD, Stripp BR: Terminal bronchioles harbor a unique airway stem cell population that localizes to the bronchoalveolar duct junction. Am J Pathol 2002, 161:173-182.

37. Xiang X, Benson KF, Chada K: Mini-mouse: disruption of the pygmy locus in a transgenic insertional mutant. Science 1990, 247:967-969.

38. Maretto S, Cordenonsi M, Dupont S, Braghetta P, Broccoli V, Hassan AB, Volpin D, Bressan GM, Piccolo S: Mapping Wnt/beta-catenin signaling during mouse development and in colorectal tumors. Proc Natl Acad SCi U S A 2003, 100:3299-3304

39. Bellusci S, Henderson R, Winnier G, Oikawa T, Hogan BL: Evidence from normal expression and targeted misexpression that bone morphogenetic protein (Bmp-4) plays a role in mouse embryonic lung morphogenesis. Development 1996, 122:1693-1702.

40. Hirning-Folz U, Wilda M, Rippe V, Bullerdiek J, Hameister H: The expression pattern of the Hmgic gene during development. Genes Chromosomes Cancer 1998, 23:350-357.

41. Tefft D, De Langhe SP, Del Moral PM, Sala F, Shi W, Bellusci S, Warburton D: A novel function for the protein tyrosine phosphatase Shp2 during lung branching morphogenesis. Dev Biol 2005, 282:422-431.

42. Del Moral PM, Sala FG, Tefft D, Shi W, Keshet E, Bellusci S, Warburton D: VEGF-A signaling through Flk-1 is a critical facilitator of early embryonic lung epithelial to endothelial crosstalk and branching morphogenesis. Dev Biol 2006, 290:177-188

43. Yates LL, Schnatwinkel C, Hazelwood L, Chessum L, Paudyal A, Hilton H, Romero MR, Wilde J, Bogani D, Sanderson J, Formstone C, Murdoch JN, Niswander LA, Greenfield A, Dean CH: Scribble is required for normal epithelial cell-cell contacts and lumen morphogenesis in the mammalian lung. Dev Biol 2013, 373:267-280.

44. Chen $\mathrm{C}$, Chen $\mathrm{H}$, Sun J, Bringas $P$ Jr, Chen $Y$, Warburton D, Shi W: Smad1 expression and function during mouse embryonic lung branching morphogenesis. Am J Physiol Lung Cell Mol Physiol 2005, 288:L1033-L1039.

45. Esquibies AE, Bazzy-Asaad A, Ghassemi F, Nishio H, Karihaloo A, Cantley LG: VEGF attenuates hyperoxic injury through decreased apoptosis in explanted rat embryonic lung. Pediatr Res 2008, 63:20-25. 
46. Barreto G, Schafer A, Marhold J, Stach D, Swaminathan SK, Handa V, Doderlein G, Maltry N, Wu W, Lyko F, Niehrs C: Gadd45a promotes epigenetic gene activation by repair-mediated DNA demethylation. Nature 2007, 445:671-675.

47. Mukhametshina RT, Ruhs A, Singh I, Hasan D, Contreras A, Mehta A, Nikam VS, Ahlbrecht K, Carraro G, Cabrera-Fuentes HA, Jiang D, Voswinckel R, Seeger W, Bellusci S, Scharffetter-Kochanek K, Bagaeva TV, Preissner KT, Boettger T, Braun T, Krüger M, Barreto G: Quantitative proteome analysis of alveolar type-Il cells reveals a connection of integrin receptor subunits beta $2 / 6$ and WNT signaling. J Proteome Res 2013, 12:5598-5608.

48. Da Huang W, Sherman BT, Lempicki RA: Systematic and integrative analysis of large gene lists using DAVID bioinformatics resources. Nat Protoc 2009, 4:44-57.

49. Edgar R, Domrachev M, Lash AE: Gene Expression Omnibus: NCBI gene expression and hybridization array data repository. Nucleic Acids Res 2002, 30:207-210

50. Konduri SD, Srivenugopal KS, Yanamandra N, Dinh DH, Olivero WC, Gujrati M, Foster DC, Kisiel W, Ali-Osman F, Kondraganti S, Lakka SS, Rao JS: Promoter methylation and silencing of the tissue factor pathway inhibitor-2 (TFPI-2), a gene encoding an inhibitor of matrix metalloproteinases in human glioma cells. Oncogene 2003, 22:4509-4516.

51. Wong B: Color blindness. Nat Methods 2011, 8:441

52. Wong B: Points of view: avoiding color. Nat Methods 2011, 8:525.

doi:10.1186/1741-7007-12-21

Cite this article as: Singh et al:: Hmga2 is required for canonical WNT signaling during lung development. BMC Biology 2014 12:21.

\section{Submit your next manuscript to BioMed Central and take full advantage of:}

- Convenient online submission

- Thorough peer review

- No space constraints or color figure charges

- Immediate publication on acceptance

- Inclusion in PubMed, CAS, Scopus and Google Scholar

- Research which is freely available for redistribution 